\title{
Theoretical Study of One-Intermediate Band Quantum Dot Solar Cell
}

\author{
Abou El-Maaty Aly ${ }^{1,2}$ and Ashraf Nasr ${ }^{2,3}$ \\ ${ }^{1}$ Power Electronics and Energy Conversion Department, Electronics Research Institute (ERI), \\ National Research Centre Building (NRCB), El-Tahrir Street, Dokki, Giza 12622, Egypt \\ ${ }^{2}$ Computer Engineering Department, College of Computer, Qassim University, P.O. Box 6688, Buryadah 51452, Saudi Arabia \\ ${ }^{3}$ Radiation Engineering Department, NCRRT, Atomic Energy Authority, P.O. Box 29, Nasr City, Egypt
}

Correspondence should be addressed to Abou El-Maaty Aly; abouelmaaty67@gmail.com

Received 3 January 2014; Accepted 3 February 2014; Published 24 March 2014

Academic Editor: Xiwang Zhang

Copyright (C) 2014 A.-M. Aly and A. Nasr. This is an open access article distributed under the Creative Commons Attribution License, which permits unrestricted use, distribution, and reproduction in any medium, provided the original work is properly cited.

\begin{abstract}
The intermediate bands (IBs) between the valence and conduction bands play an important role in solar cells. Because the smaller energy photons than the bandgap energy can be used to promote charge carriers transfer to the conduction band and thereby the total output current increases while maintaining a large open circuit voltage. In this paper, the influence of the new band on the power conversion efficiency for the structure of the quantum dots intermediate band solar cell (QDIBSC) is theoretically investigated and studied. The time-independent Schrödinger equation is used to determine the optimum width and location of the intermediate band. Accordingly, achievement of maximum efficiency by changing the width of quantum dots and barrier distances is studied. Theoretical determination of the power conversion efficiency under the two different ranges of QD width is presented. From the obtained results, the maximum power conversion efficiency is about $70.42 \%$ for simple cubic quantum dot crystal under full concentration light. It is strongly dependent on the width of quantum dots and barrier distances.
\end{abstract}

\section{Introduction}

The intermediate band solar cells (IBSCs) have attracted great attention due to the possibility of exceeding the ShockleyQueisser (SQ) limit [1-4]. From the analysis by Luque et al. $[5,6]$, the IBSC's concept yields a maximum theoretical efficiency of $63.2 \%$, surpassing the limit of $40.7 \%$ of single gap solar cells under maximum light concentration (the sun being assumed as a blackbody at $6000 \mathrm{~K}$ ) [7]. Since its introduction in 1997, there have been many theoretical and experimental efforts to explore this idea [8]. The use of quantum dot (QD) technology is proposed as a near term proof of concept of the operating principles of an intermediate band solar cell (IBSC). This intermediate band allows the extra generation of electron-hole pairs through the two-step absorption of subbandgap photons. In the first step, an electron is pumped from the valence band (VB) to the intermediate band (IB), while in the second step, another electron is launched from the IB to the CB [9]. Quantum dot heterojunctions may implement an IBSC because of their ability to provide the three necessary bands [10]. In comparison to conventional quantum well superlattices or multiple quantum well structures, quantum dot superlattice (QDS) that consists of multiple arrays of quantum dots has many advantages due to its modified density of electronic states and optical selection rules. For example, due to relaxed intraband optical selection rules in QDS, they are capable of absorbing normally incident radiation, for example, Indium gallium nitride $\left(\operatorname{In}_{x} \mathrm{Ga}_{1-x} \mathrm{~N}\right)$ alloys feature a bandgap ranging from the near infrared $(\sim 0.7 \mathrm{eV})$ to ultraviolet $(\sim 3.42 \mathrm{eV})$; this range corresponds very closely to the solar spectrum, making $\operatorname{In}_{x} \mathrm{Ga}_{1-x} \mathrm{~N}$ alloys a promising material for future solar cells. $\operatorname{In}_{x} \mathrm{Ga}_{1-x} \mathrm{~N}$ alloys solar cells have been fabricated with different indium contents and the results are encouraging [11]. However, it is not possible in quantum well superlattices. The latter makes QDS a good candidate for infrared photodetector applications [12]. As considered in [13], 3D-ordered QDS with the closely spaced quantum 
dots and high quality interfaces allow a strong wave function overlap and the formation of minibands. In such structures, the quantum dots play a similar role to that of atoms in real crystals. To distinguish such nanostructures from the disordered multiple arrays of quantum dots, we refer to them as quantum dot supracrystals (QDC). Other assumptions that include the rules for solving the Schrödinger equation for determining the IBs are considered in the following sections. The remainder of the paper is organized as follows. In Section 2, the basic assumptions and a superlattice model description are presented. The intermediate band energy and wavevectors of a charge carrier are calculated in Section 3. The induced photocurrent density and power conversion energy are described in Section 4. The numerical results and discussions are summarized in Section 5. Finally, a conclusion of the results is outlined in Section 5.

\section{Basic Assumptions and Superlattice Model Description}

In this paper, the investigations are devoted to one intermediate band solar cell. We explain the various behaviors of this model depending on the QD solar cell parameters, such as quantum dot size, interdot distance, and type of composition alloy. Meanwhile, in the next step, the multi-intermediate bands are investigated. Therefore, the following are the basic assumptions that are used in the QD one intermediate band solar cell model [11-14].

(a) The solar cell model is thick enough; carrier mobility will be high enough to ensure the full absorption of photons. All photons with energy greater than the lowest energy gap in the QDIBS model are absorbed.

(b) The quasi-Fermi energy levels which are equivalent to infinite carrier mobility are constant throughout the model.

(c) The transitions occurring between the bands are only radiative recombination.

(d) The solar cell absorbs blackbody radiation at a sun temperature, $6000 \mathrm{~K}$, and the temperature of the solar cell, $300 \mathrm{~K}$, and emits blackbody radiation at $300 \mathrm{~K}$ only.

(e) No carriers can be extracted from the intermediate band; the net pumping of electrons from the VB to the IB must equal the net pumping of electrons from the IB to the CB.

(f) The shape of the QDs is cubic and they must be arranged in a periodic lattice in order to establish well-placed intermediate band boundaries. For simplicity, the energy corresponding to the top of the valence band is the same both in the barrier and the QD material; therefore there is no valence band offset and only confining potential occurs at the conduction band offset. (g) The intermediate band should be approximately halffilled with electrons in order to receive electrons from the valence energy band and pump electrons to the conduction energy band.

When charge carriers in semiconductors can be confined by potential barriers in three dimensions, it is called quantum dots. QDs periodic arrays from semiconductor with a smaller bandgap are sandwiched between two layers of another second semiconductor having a larger bandgap (n or $\mathrm{p}$ type). This configuration creates the potential barriers. Two potential wells are formed in this structure; one is for conduction band electrons and the other for valence band holes. The well (QDs layer) depth for electrons is called the conduction band offset, which is the difference between the conduction band edges of the well and barrier semiconductors. The well depth for holes is called the valence band offset. If the offset for either the conduction or valence bands is zero, then only one carrier will be confined in a well. In this structure, if the barrier thickness between adjacent wells prevents significant electronic coupling between the wells, then each well is electronically isolated. On the other hand, if the barrier thickness is sufficiently thin to allow electronic coupling between wells, then the electronic charge distribution can become delocalized along the direction normal to the well layers, therefore producing new minibands (see Figure 1).

The electronic coupling rapidly increases with decreasing the barrier thickness and miniband formation is very strong below $2 \mathrm{~nm}$ [15]. Superlattice structures yield efficient charge transport normal to the layers because the charge carriers can move through the minibands. As a result the barrier will be narrower and the miniband and the carrier mobility will be wider and higher, respectively.

\section{Wavevectors of a Charge Carrier}

The wavevector of a charge carrier (single electron or hole) can be described by the time-independent Schrödinger equation, which has the following form $[11,16]$ :

$$
\left(\frac{-\hbar^{2}}{2 m} \nabla^{2}+V\right) k=E k
$$

where $\hbar$ is the Plank's constant, $m$ is the effective mass, $\nabla^{2}$ is a second order differential operator, $V$ is the potential energy, $E$ is the total energy of charge carrier, and $k$ is the wavevector. The Kronig-Penney model solved this equation by the onedimensional periodic potential shown also in Figure 1.

This model assumes that the wave travelling of charge carrier is in the positive $x$ direction for one-dimensional only. Therefore, the mathematical form of the repeating unit of the potential is

$$
V(x)= \begin{cases}V_{0}, & \text { for } x=L_{B}, \\ 0, & \text { for } x=L_{\mathrm{QD}}\end{cases}
$$

Here, $V_{0}$ is the conduction band offset, $L_{B}$ is the barrier width, and $L_{\mathrm{QD}}$ is the quantum dot width. The period $T$ of the 


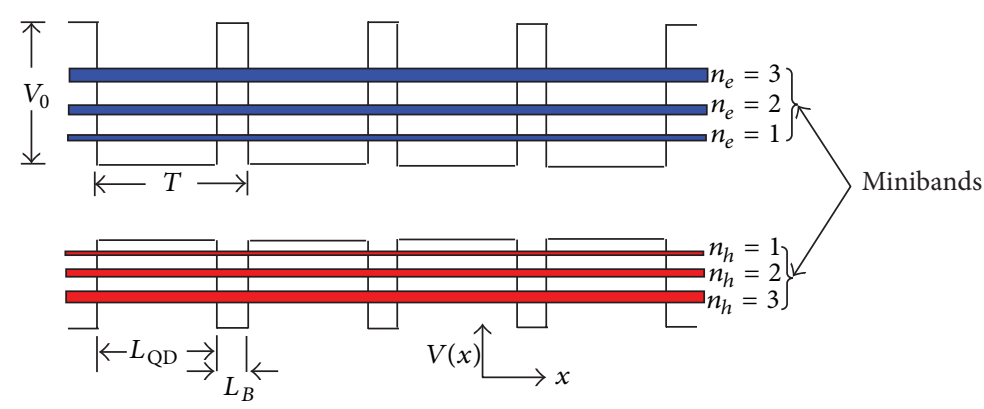

Figure 1: Minibands formation in the superlattice structure and Kronig-Penney potential.

considered potential is equal to $\left(L_{\mathrm{QD}}+L_{B}\right)$. The Schrödinger equation for this model is $[10,17,18]$.Consider

$$
\begin{gathered}
\frac{d^{2} k}{d x^{2}}+\frac{2 m E}{\hbar^{2}} k=0, \quad \text { for } x=L_{\mathrm{QD}}, \\
\frac{d^{2} k}{d x^{2}}+\frac{2 m\left(E-V_{0}\right)}{\hbar^{2}} k=0, \quad \text { for } x=L_{B} .
\end{gathered}
$$

According to the Kronig-Penney model, the solution of (3a) and ( $3 b)$ can be expressed as

$$
\begin{aligned}
& \frac{\sigma^{2}-\delta^{2}}{2 \sigma \delta} \sinh \left(\sigma L_{B}\right) \sin \left(\delta L_{\mathrm{QD}}\right)-\cosh \left(\sigma L_{B}\right) \cos \left(\delta L_{\mathrm{QD}}\right) \\
& \quad=\cos \left(L_{B}+L_{\mathrm{QD}}\right) k .
\end{aligned}
$$

For simplicity, one can assume the following symbols for internal terms in (4):

$$
\begin{gathered}
\sigma^{2}=\frac{2 m_{B}\left(V_{0}-E\right)}{\hbar^{2}}=\frac{2 m_{B} V_{0}}{\hbar^{2}}(1-\epsilon), \\
\delta^{2}=\frac{2 m_{Q} V_{0}}{\hbar^{2}} \frac{E}{V_{0}}=\frac{2 m_{Q} V_{0}}{\hbar^{2}} \epsilon, \quad \epsilon=\frac{E}{V_{0}},
\end{gathered}
$$

where $m_{B}, m_{Q}$ are effective mass of electron in barrier region and effective mass of electron in quantum dots region, respectively.

Therefore, from (4), the factor into the first term plays an important role for investigating this proposed QDIBSC model. It can be expressed as follows:

$$
\frac{\sigma^{2}-\delta^{2}}{2 \sigma \delta}=\frac{m_{\mathrm{Q}}-\left(m_{\mathrm{Q}}+m_{B}\right) \epsilon}{2\left(m_{\mathrm{Q}} m_{B} \epsilon\right)^{1 / 2}(\epsilon-1)^{1 / 2}} .
$$

Furthermore, the other arguments in (4) for hyperbolic and sinusoidal functions can be defined as

$$
\begin{gathered}
\sigma L_{B}=\mu A_{B}(1-\epsilon)^{1 / 2}, \quad \delta L_{\mathrm{QD}}=A_{\mathrm{Q}} \epsilon^{1 / 2}, \\
\mu=\frac{L_{B}}{L_{\mathrm{QD}}}, \quad A_{B}=L_{\mathrm{QD}}\left(\frac{2 m_{B} V_{0}}{\hbar^{2}}\right)^{1 / 2}, \\
A_{\mathrm{Q}}=L_{\mathrm{QD}}\left(\frac{2 m_{\mathrm{Q}} V_{0}}{\hbar^{2}}\right)^{1 / 2} .
\end{gathered}
$$

Substituting these definitions into (4), it will become as

$$
\begin{gathered}
\frac{m_{\mathrm{Q}}-\left(m_{\mathrm{Q}}+m_{B}\right) \epsilon}{2\left(m_{\mathrm{Q}} m_{B} \epsilon\right)^{1 / 2}(1-\epsilon)^{1 / 2}} \sinh \left[\mu A_{B}(1-\epsilon)^{1 / 2}\right] \sin \left(A_{\mathrm{Q}} \epsilon^{1 / 2}\right) \\
\quad+\cosh \left[\mu A_{B}(1-\epsilon)^{1 / 2}\right] \cos \left(A_{\mathrm{Q}} \epsilon^{1 / 2}\right) \\
=\cos \left[k L_{\mathrm{QD}}(1+\mu)\right], \quad \text { for } \epsilon<1,
\end{gathered}
$$

$$
\begin{aligned}
& \frac{m_{\mathrm{Q}}-\left(m_{\mathrm{Q}}+m_{B}\right) \epsilon}{2\left(m_{\mathrm{Q}} m_{B} \epsilon\right)^{1 / 2}(\epsilon-1)^{1 / 2}} \sin \left[\mu A_{B}(\epsilon-1)^{1 / 2}\right] \\
& \quad \times \sin \left(A_{\mathrm{Q}} \epsilon^{1 / 2}\right)+\cos \left[\mu A_{B}(\epsilon-1)^{1 / 2}\right] \cos \left(A_{\mathrm{Q}} \epsilon^{1 / 2}\right) \\
& =\cos \left[k L_{\mathrm{QD}}(1+\mu)\right], \quad \text { for } \epsilon>1,
\end{aligned}
$$

$$
\cos A_{\mathrm{Q}}-\frac{\mu A_{B}}{2} \sin A_{\mathrm{Q}}=\cos \left[k L_{\mathrm{QD}}(1+\mu)\right]
$$

$$
\text { for } \epsilon=1 \text {. }
$$

The left-hand side of (8a), (8b), and (8c) can be represented by $F(\epsilon)$ for all values of the ratio of total energy of electrons over conduction band offset: $\left(\epsilon=E / V_{0}\right)$. Consider

$$
F(\epsilon)=\cos \left[k L_{\mathrm{QD}}(1+\mu)\right] .
$$

Equation (9) cannot be solved analytically, but it can be solved graphically. Figure 2 shows the left-hand side of (9), $F(\epsilon)$, against $\epsilon$ at fixed values of $\mu$ and $A$. The concerned QDIBSC model depends on the considered alloys in previous studies for comparing the results; the quantum dot width: $L_{\mathrm{QD}}=$ $4.5 \mathrm{~nm}\left(\operatorname{InAs}_{0.9} \mathrm{~N}_{0.1}, m_{\mathrm{Q}}=0.0354 m_{0}\right)$, barrier width: $L_{B}=$ $2 \mathrm{~nm}\left(\mathrm{GaAs}_{0.98} \mathrm{Sb}_{0.02}, m_{B}=0.066 m_{0}\right)$, and $V_{0}=1.29 \mathrm{eV}$ [12]. But in further studies, other alloys will be processed. The lefthand side is not constrained to \pm 1 and is a function of energy only. The right-hand side is constrained to a range of \pm 1 and is a function of $k$ only. The limits of the right-hand side \pm 1 occur at $k=0$ to $( \pm \pi / T)$. The two horizontal red lines in Figure 2 represent the two extreme values of $\cos \left[k L_{\mathrm{QD}}(1+\mu)\right]$. The only allowed values of $E$ are those for which $F(\epsilon)$ lies between the two horizontal red lines; see Table 1.

Figure 2 and Table 1 show the allowed energy ranges or bands, $E$, which fall into continuous regions separated by 
TABLE 1: The allowed energy ranges or bands as $E$ for the proposed composition from Figure 2.

\begin{tabular}{lc}
\hline Band & Range of $E,(\mathrm{eV})$ \\
\hline 1 & $0.2039-0.2369$ \\
2 & $0.8140-0.9852$ \\
3 & $1.6638-2.2775$ \\
4 & $2.4758-3.5439$ \\
\hline
\end{tabular}

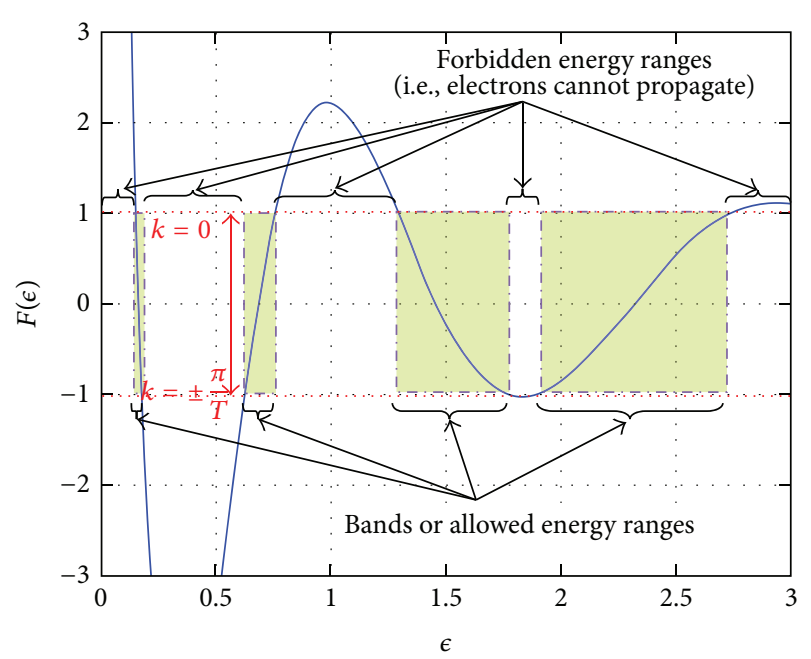

FIgURE 2: The left-hand side of (9), ( $\epsilon$ ), against $\epsilon$ at fixed values of $\mu$ and $A$. This graph shows allowed and forbidden electron energy bands for the simple cubic $\mathrm{QD}$ with the quantum dot width $L_{\mathrm{QD}}=$ $4.5 \mathrm{~nm}\left(\operatorname{InAs}_{0.9} \mathrm{~N}_{0.1}, m_{\mathrm{Q}}=0.0354 m_{0}\right)$, barrier width $L_{B}=2 \mathrm{~nm}$ $\left(\mathrm{GaAs}_{0.98} \mathrm{Sb}_{0.02}, m_{B}=0.066 m_{0}\right)$, and $V_{0}=1.29 \mathrm{eV}$.

gaps. This distribution of allowed energies illustrates the band structure of crystalline solids. According to Figure 2 and Table 1, when the values of $E$ are from 0 to $0.2039 \mathrm{eV}, F(\epsilon)$ is greater than one and so there is no real value of $k$ that satisfies (9). At $E=0.2039 \mathrm{eV}$ the wavevector will be equal to zero. As $E$ varies from $0.2039 \mathrm{eV}$ to $0.2369 \mathrm{eV}, \cos \left[k L_{\mathrm{QD}}(1+\mu)\right]$ varies from +1 to -1 and $k L_{\mathrm{QD}}(1+\mu)$ varies from 0 to $( \pm \pi)$. As $E$ varies from $0.2369 \mathrm{eV}$ to $0.8140 \mathrm{eV}$, there is no real value of $k$ that satisfies (9). At $E=0.8140 \mathrm{eV}$, the argument of the righthand side, $k L_{\mathrm{QD}}(1+\mu)$, will be equal to $(\pi)$. At $E=0.9852 \mathrm{eV}$, the argument of the right-hand side, $k L_{\mathrm{QD}}(1+\mu)$, will be equal to $(2 \pi)$. Other bandgaps will periodically appear with the same behavior.

Figure 3 shows the allowed energy bands and the wavevector states in one-dimensional crystal with the same data as in Figure 2. In this case, the wavevector is changed from 0 to $( \pm \pi / T)$, and the allowed energy bands into the electron energy is assigned. Also, the curve shows that the slope $(d E / d k)$ is zero at the $k$ boundaries (i.e., 0 and $\pm \pi / T)$. Thus, the velocity of the electrons approaches zero at the boundaries. This means that the electron trajectory or momentum is confined to stay within the allowable $k$. Figure 4 denotes more explanation for the considered energy allowed bands as in Figure 3. It shows the allowed $E-k$ states with the bandgaps that appear when $k=N \pi / T$. The

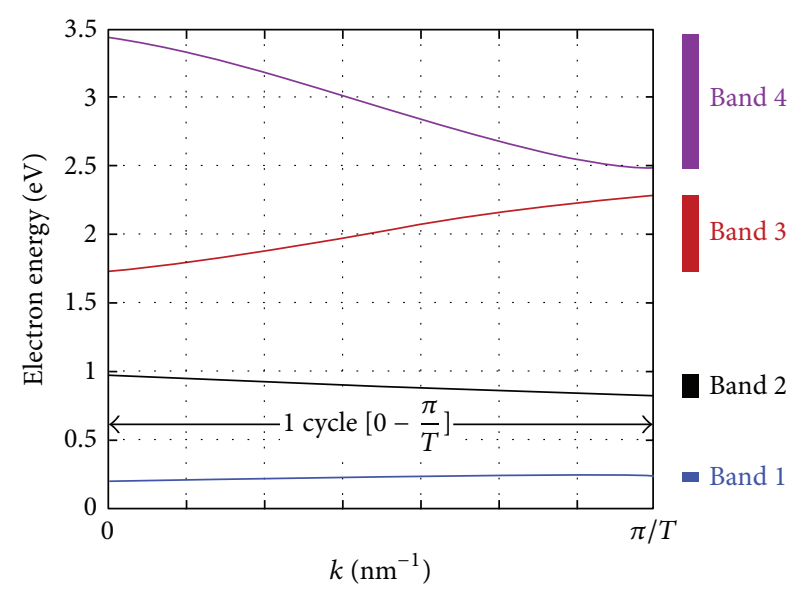

FIgURE 3: Allowed $E-k$ states in one-dimensional crystal with the same data in Figure 2.

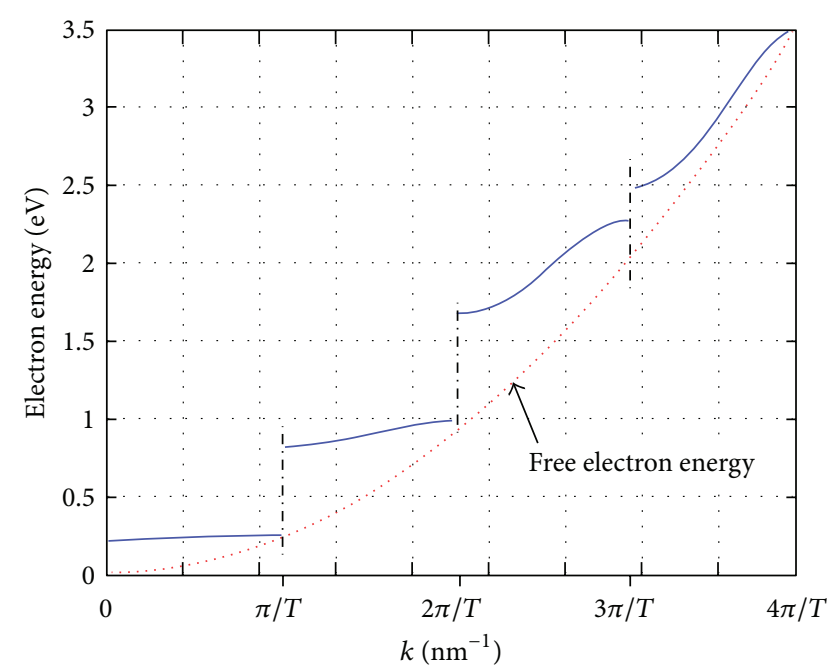

FIgURE 4: Allowed $E-k$ states and free electrons energy.

dashed line represents $E-k$ states for free electrons energy. It can be obtained by letting $V_{0}=0$ in (4). Therefore, the free energy of electron is $E=\hbar^{2} k^{2} / 2 m$. After the essential equations and assumptions are demonstrated, the following section is concerned with the determination of the effect of intermediate band, alloy construction, and dot and barrier width in each of induced photocurrent density and corresponding power efficiency.

\section{Induced Current Density and Power Conversion Efficiency}

The photon generated current density in QDIBSC with one intermediate band is derived in this section. Then, the sensitivity of the power conversion efficiency as a function of the intermediate band energy level will be investigated. According to the assumptions in Section 2, the only radiative transitions occur between the bands therefore the generation 


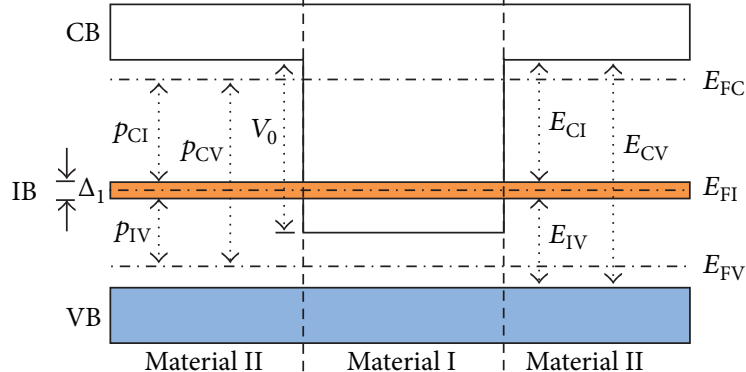

Figure 5: Construction of energy band diagram for two semiconductor materials (material I; InAs ${ }_{0.9} \mathrm{~N}_{0.1}$ : quantum dot, material II; $\mathrm{GaAs}_{0.98} \mathrm{Sb}_{0.02}$ : barrier width) to create a heterostructure for one intermediate band solar cell.

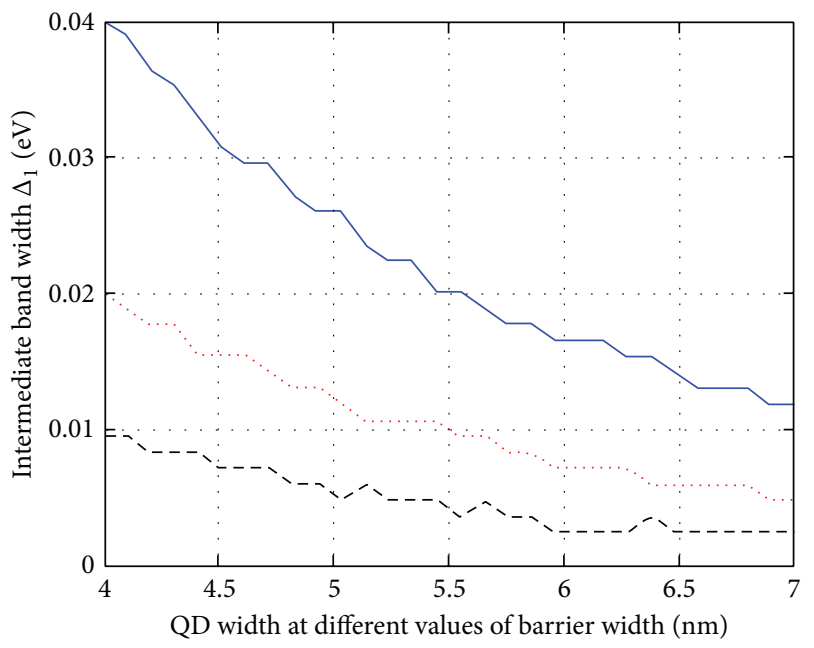

$$
\begin{aligned}
& \text { Barrier width: } \\
& ---2(\mathrm{~nm}) \\
& \cdots \cdots 2.5(\mathrm{~nm}) \\
& -3(\mathrm{~nm})
\end{aligned}
$$

FIGURE 6: Change width of intermediate band $\left(\Delta_{1}\right)$ with change QD width for the first allowable range at three different values of barrier width.

and recombination events are represented by photon absorption and emission. Figure 5 illustrates the construction of an energy band diagram for a heterostructure in the case of one intermediate band solar cell. An electron in the valence band can be excited to either the intermediate or conduction band. Also, an electron in the intermediate band can be excited to the conduction band. Therefore, there are three upward energy transitions: $E_{\mathrm{VI}}, E_{\mathrm{CI}}$, and $E_{\mathrm{CV}} \cdot E_{\mathrm{VI}}$ represents valence to intermediate band, $E_{\mathrm{CI}}$ represents intermediate to conduction band, and $E_{\mathrm{CV}}$ represents the conventional bandgap between the valence and conduction band. The two intermediate transitions $E_{\mathrm{VI}}$ and $E_{\mathrm{CI}}$ are independent of each other, while the bandgap transition $E_{\mathrm{CV}}$ is a function of the two intermediate ones: $E_{\mathrm{CV}}=E_{\mathrm{VI}}+E_{\mathrm{CI}}$.

The photon flux density, $N$, is the number of photons per second per unit area per unit wavelength and behaves like a blackbody flux density and according to the RoosbroeckShockley equation [19-22] is given by

$$
N\left(E_{\mathrm{VI}}, E_{\mathrm{CI}}, T, p\right)=\frac{2 \pi \xi}{h^{3} C^{2}} \int_{E_{\mathrm{VI}}}^{E_{\mathrm{CI}}} \frac{E^{2} d E}{e^{(E-p) / k_{B} T}-1},
$$

where $T$ is the temperature, $\xi$ is geometric factor, $h$ is Plank constant, $k_{B}$ is Boltzmann constant, $C$ is speed of light, and $p$ is chemical potential. To simplify the analysis, assume that $E_{\mathrm{VI}} \leq E_{\mathrm{CI}} \leq E_{\mathrm{CV}}$. Therefore the photons with energy greater than $E_{\mathrm{VI}}$ and less than $E_{\mathrm{CI}}$ are absorbed and electrons transfer from valence band to intermediate band and leave holes in the valence band. Any excess energy greater than $E_{\mathrm{VI}}$ and less than $E_{\mathrm{CI}}$ will be lost due to thermalization and carriers will relax to the band edges before another radiative occurs. One can notice that this thermalization value is very small in comparison with its value in the case of bulk based semiconductor solar cells. This means that an absorbed photon with energy greater than $E_{\mathrm{VI}}$ and less than $E_{\mathrm{CI}}$ has the same effect as an absorbed photon with energy equal to $E_{\mathrm{VI}}$.

Photons with energy greater than $E_{\mathrm{CI}}$ and less than $E_{\mathrm{CV}}$ are absorbed and an electron transfers from intermediate band to conduction band. The excess energy behavior has the same effect as considered in the previous case regarding the difference in the transition band process. Photons with energy greater than $E_{\mathrm{CV}}$ are absorbed and an electron transfers from valence band to conduction band and creates a hole in the valence band. The absorbed photon with energy greater than $E_{\mathrm{CV}}$ has the same effect as an absorbed photon with energy equal to $E_{C V}$ taking into account the excess energy process. The net photon flux is equal to the number of charge carrier flux collected at the contact. When the charge carrier flux is multiplied by the electric charge of electron, $q$, the current density of the QDIBSC for one intermediate band is $[11,13,14]$ :

$$
\begin{aligned}
j=q\{ & {\left[C_{f} \xi N\left(E_{\mathrm{CV}}, \infty, T_{s}, 0\right)\right.} \\
+ & \left(1-C_{f} \xi\right) N\left(E_{\mathrm{CV}}, \infty, T_{a}, 0\right) \\
& \left.-p N\left(E_{\mathrm{CV}}, \infty, T_{a}, q V\right)\right] \\
+ & {\left[C_{f} \xi N\left(E_{\mathrm{CI}}, E_{\mathrm{CV}}, T_{s}, 0\right)\right.} \\
& +\left(1-C_{f} \xi\right) N\left(E_{\mathrm{CI}}, E_{\mathrm{CV}}, T_{a}, 0\right) \\
& \left.\left.-N\left(E_{\mathrm{CI}}, E_{\mathrm{CV}}, T_{a}, p_{\mathrm{CI}}\right)\right]\right\},
\end{aligned}
$$

where $C_{f}$ is concentration factor, $T_{s}$ is temperature of sun $(6000 \mathrm{~K}), T_{a}$ is ambient temperature $(300 \mathrm{~K}), q V$ is quasiFermi energy, and $p_{\mathrm{CI}}$ is chemical potential between conduction and intermediate bands. The terms in the first bracket represent the current density generated when the electrons transfer from the valence band to the conduction band as typically for conventional solar cell. While the terms in the second bracket represent the current density generated when the electrons transfer from the intermediate band to the conduction band. In both bracketed terms, the QDIBSC absorbs radiation from the sun at the temperature $T_{s}$ and 


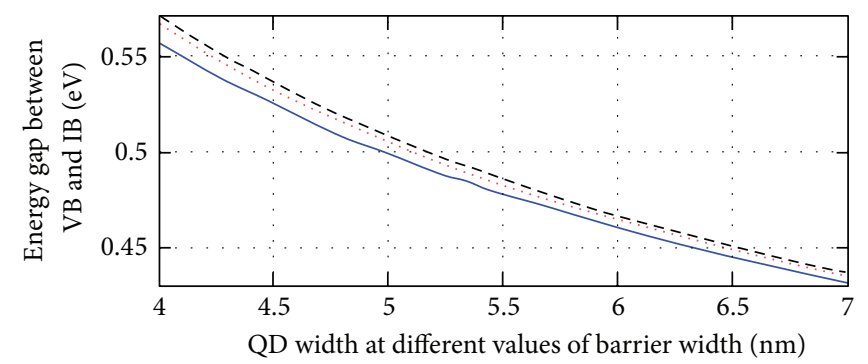

Barrier width:

$---2(\mathrm{~nm})$

$2.5(\mathrm{~nm})$

$3(\mathrm{~nm})$

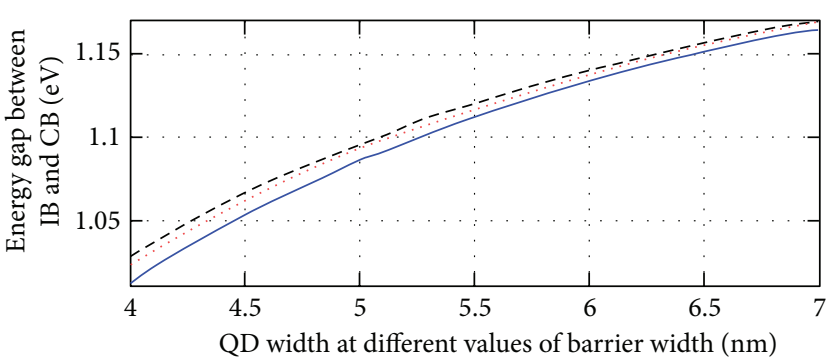

Barrier width:

- - 2 (nm)

$2.5(\mathrm{~nm})$

$3(\mathrm{~nm})$

(a)

(b)

FIGURE 7: (a) Change energy gap between VB and IB according to change in QD width for the first allowable range at different values of barrier width. (b) Change energy gap between IB and CB with the same conditions in (a).

$T_{a}$, respectively, while it emits radiation at the temperature $T_{a}$ and a corresponding chemical potential. The current density of the QDIBSC is formulated according to the proper operation of the QDIBSC which requires that there is no current extracted from the intermediate band; that is, the current entering the intermediate band must equal the current leaving the intermediate band. Therefore, the second term in (11) can be rewritten as $[14,23,24]$

$$
\begin{aligned}
q\left[C_{f} \xi N\left(E_{\mathrm{CI}}, E_{\mathrm{CV}}, T_{s}, 0\right)+\left(1-C_{f} \xi\right)\right. \\
\left.\times N\left(E_{\mathrm{CI}}, E_{\mathrm{CV}}, T_{a}, 0\right)-N\left(E_{\mathrm{CI}}, E_{\mathrm{CV}}, T_{a}, p_{\mathrm{CI}}\right)\right] \\
=q\left[C_{f} \xi N\left(E_{\mathrm{VI}}, E_{\mathrm{CI}}, T_{s}, 0\right)+\left(1-C_{f} \xi\right)\right. \\
\left.\quad \times N\left(E_{\mathrm{VI}}, E_{\mathrm{CI}}, T_{a}, 0\right)-N\left(E_{\mathrm{VI}}, E_{\mathrm{CI}}, T_{a}, p_{\mathrm{IV}}\right)\right] .
\end{aligned}
$$

The output voltage can be described as the difference of the chemical potentials between $\mathrm{CB}$ and VB; that is, $q V_{\mathrm{oc}}=p_{\mathrm{CV}}=$ $p_{\mathrm{CI}}+p_{\mathrm{IV}}$.

In this work, the light intensity on QDIBSC is calculated by the number of suns, where 1 sun (or concentration factor $C_{f}=1$ ) means the standard intensity at the surface of the Earth's atmosphere. Therefore, at the surface of the Earth's atmosphere the power density falling on a QDIBSC is $P_{\text {in }}=\xi \sigma_{s} T_{s}^{4}=1587.2 \mathrm{w} / \mathrm{m}^{2}$, where $\sigma_{s}$ is Stefan's constant and $T_{s}$ is temperature of sun $(6000 \mathrm{~K})$. Theoretically, the full concentration would be achieved when $C_{f}=1 / \xi=$ 46296. The power conversion efficiency, $\eta$, of the QDIBSC is dependent on $P_{\text {in }}$, so that it varies with the level concentration of $C_{f}$. We concentrate our study on the QDIBSC efficiencies with unconcentrated light $C_{f}=1$ and also compared it with full concentration light $C_{f} \times \xi=1$. The power conversion efficiency equation of the QDIBSC is

$$
\eta=\frac{V_{\mathrm{oc}} \times J_{\mathrm{SC}} \times F F}{P_{\mathrm{in}}}=\frac{J_{m} \times V_{m}}{C_{f} \xi \sigma_{s} T_{s}^{4}}=\frac{J_{m} \times V_{m}}{C_{f} \times 1587.2},
$$

where $V_{\text {oc }}$ is open circuit voltage, $J_{\text {sc }}$ is short circuit current density, $F F$ is fill factor, $V_{m}$ is maximum voltage of the QDIBSC, and $J_{m}$ is maximum current density of the QDIBSC. After mathematical concepts and assumptions are defined in previous sections for assigned composition of material, the following section reports some features of the QDIBSCs performance for one intermediate band case.

\section{Numerical Results and Discussions}

The discussion firstly starts by manifesting the relation between QDIBSC parameters and the distribution of IB energies in the gap between valence and conduction bands. When the Schrödinger equation (1) is solved, many solutions are obtained. Some of them can be satisfied but others cannot. From the satisfied solutions, there are multi-intermediate bands as pointed from Figures 2, 3, and 4. They are essentially dependent on QDIBSC parameters such as QD, barrier widths. Here, we are concerned only with the effect of the first intermediate band, IB, into the behavior of the proposed model. From the obtained results, we found two ranges of quantum dot widths that vary the positions of intermediate bands. Other ranges of quantum dot widths will denote unachievable behavior. In the following discussions, the energy distributions, $J-V$ characteristics, and corresponding power conversion efficiency are studied for each range. Comparisons between the two ranges outcomes are processed. A summary of all the related values describing the model is considered in Table 2. For the first range (QD width changes from $4-7 \mathrm{~nm}$ ), the dependence of first intermediate band width, $\Delta_{1}$, on QD width at different barrier widths is depicted in Figure 6. One can recognize that the width of $\mathrm{IB}, \Delta_{1}$, is decreased with an increase in the QD width. This behavior is consistent with the previous investigations that demonstrate that the decreasing of $\Delta_{1}$ IB will assist in the fall down of the power conversion efficiency, as will be described later $[11,14]$. Also the same trend will be found in the second range of QD and barrier widths, which also denotes the first IB. Also, Figure 6 illustrates that when the barrier width increases in 
TABLE 2: Three different values of QD width $\left(\operatorname{InAs}_{0.9} \mathrm{~N}_{0.1}\right)$ and barrier width $\left(\mathrm{GaAs}_{0.98} \mathrm{Sb}_{0.02}\right)$ for two different ranges of QD available widths at full concentration and unconcentration.

\begin{tabular}{|c|c|c|c|c|c|c|c|c|c|c|c|c|c|c|}
\hline \multirow{2}{*}{$\begin{array}{l}\text { Two available } \\
\text { ranges }\end{array}$} & \multicolumn{2}{|c|}{ Parameters } & \multicolumn{6}{|c|}{ Full concentration } & \multicolumn{6}{|c|}{ Unconcentration } \\
\hline & $\begin{array}{l}L_{\mathrm{QD}} \\
(\mathrm{nm}) \\
\end{array}$ & $\begin{array}{c}L_{B} \\
(\mathrm{~nm})\end{array}$ & $\begin{array}{r}\eta_{\max } \\
(\%)\end{array}$ & $\begin{array}{c}V_{\max } \\
(\mathrm{v})\end{array}$ & $\begin{array}{c}J_{\max } \times \xi \\
\left(\mathrm{mA} / \mathrm{cm}^{2}\right)\end{array}$ & $V_{\mathrm{oc}}(\mathrm{v})$ & $\begin{array}{c}J_{\mathrm{sc}} \times \xi \\
\left(\mathrm{mA} / \mathrm{cm}^{2}\right)\end{array}$ & FF & $\begin{array}{r}\eta_{\max } \\
(\%)\end{array}$ & $\begin{array}{c}V_{\max } \\
(\mathrm{v})\end{array}$ & $\begin{array}{c}J_{\max } \\
\left(\mathrm{mA} / \mathrm{cm}^{2}\right)\end{array}$ & $\begin{array}{l}V_{\mathrm{oc}} \\
(\mathrm{v})\end{array}$ & $\begin{array}{c}J_{\mathrm{sc}} \\
\left(\mathrm{mA} / \mathrm{cm}^{2}\right)\end{array}$ & $\mathrm{FF}$ \\
\hline \multirow{6}{*}{$\begin{array}{l}\text { "1st range" } \\
4-7 \mathrm{~nm}\end{array}$} & 4 & 2 & 63.2 & 1.503 & 66.8 & 1.57 & 67.9 & 0.942 & 51.6 & 1.231 & 66.5 & 1.33 & 67.9 & 0.906 \\
\hline & 4.5 & 2 & 61.2 & 1.505 & 64.6 & 1.58 & 65.6 & 0.938 & 50.0 & 1.233 & 64.3 & 1.34 & 65.6 & 0.902 \\
\hline & 5 & 2 & 59.6 & 1.507 & 62.8 & 1.58 & 63.8 & 0.939 & 48.7 & 1.235 & 62.5 & 1.34 & 63.8 & 0.903 \\
\hline & 4 & 2 & 63.2 & 1.503 & 66.8 & 1.57 & 67.9 & 0.942 & 51.6 & 1.231 & 66.5 & 1.33 & 67.9 & 0.906 \\
\hline & 4 & 2.5 & 63.1 & 1.514 & 66.2 & 1.58 & 67.3 & 0.943 & 51.6 & 1.242 & 65.9 & 1.34 & 67.3 & 0.908 \\
\hline & 4 & 3 & 63.0 & 1.516 & 65.9 & 1.58 & 67.0 & 0.944 & 51.5 & 1.244 & 65.7 & 1.35 & 67.0 & 0.904 \\
\hline \multirow{6}{*}{$\begin{array}{l}\text { "2nd range" } \\
7-11 \mathrm{~nm}\end{array}$} & 8.1 & 1.98 & 70.4 & 1.508 & 74.1 & 1.57 & 75.3 & 0.945 & 57.5 & 1.238 & 73.7 & 1.34 & 75.3 & 0.904 \\
\hline & 8.6 & 1.98 & 68.4 & 1.510 & 71.9 & 1.57 & 73.1 & 0.946 & 55.9 & 1.238 & 71.6 & 1.34 & 73.1 & 0.905 \\
\hline & 9.1 & 1.98 & 66.7 & 1.512 & 70.0 & 1.58 & 71.2 & 0.941 & 54.5 & 1.241 & 69.8 & 1.34 & 71.2 & 0.908 \\
\hline & 8.1 & 1.98 & 70.4 & 1.508 & 74.1 & 1.57 & 75.3 & 0.945 & 57.5 & 1.238 & 73.7 & 1.34 & 75.3 & 0.904 \\
\hline & 8.1 & 2.48 & 70.3 & 1.519 & 73.4 & 1.58 & 74.7 & 0.945 & 57.5 & 1.248 & 73.1 & 1.35 & 74.6 & 0.906 \\
\hline & 8.1 & 2.98 & 70.2 & 1.522 & 73.2 & 1.59 & 74.4 & 0.942 & 57.4 & 1.250 & 72.9 & 1.35 & 74.4 & 0.907 \\
\hline
\end{tabular}

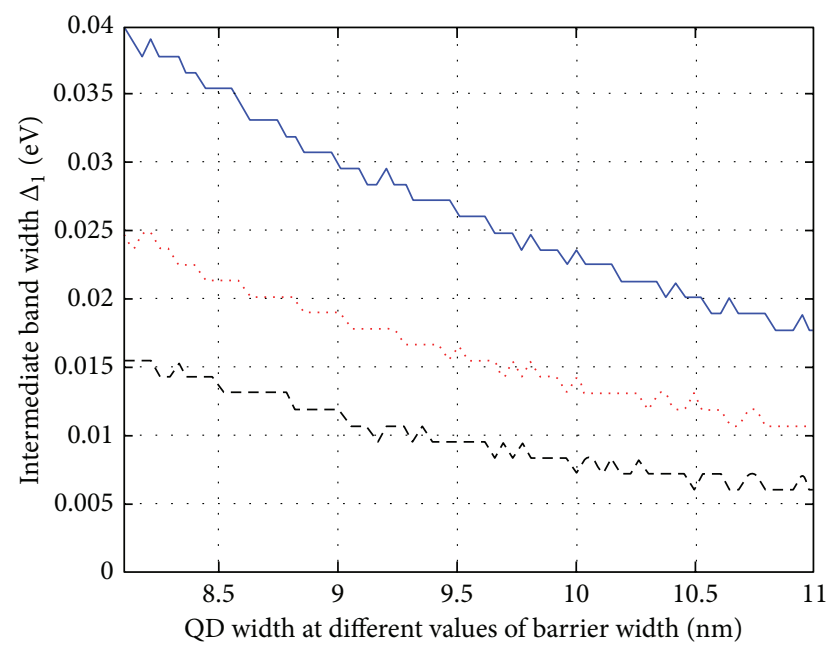

Barrier width:

- - $1.98(\mathrm{~nm})$

… $2.34(\mathrm{~nm})$

$2.7(\mathrm{~nm})$

FIGURE 8: Change width of intermediate band $\left(\Delta_{1}\right)$ with change QD width for the second allowable range at three different values of barrier width.

the allowable range $(2-3 \mathrm{~nm}), \Delta_{1}$ IB somewhat increases. At the same time, the increase of barrier width will somewhat inversely decrease the energy gap between IB width and each of $\mathrm{VB}$ and $\mathrm{CB}$, as this behavior is shown in Figures 7(a) and 7 (b). It is expected that when $\Delta_{1}$ IB enlarges, the energy gap difference between it and the considered $\mathrm{VB}$ and $\mathrm{CB}$ will be decreased, as the total distance $E_{\mathrm{cv}}$ is constant $[11,14]$. The first range power efficiency and $J-V$ characteristics will be compared with the second range in the last part of these discussions.
For the second allowable range (QD width changes from $8.1-9.1 \mathrm{~nm})$ and at the same barrier width range $(2-3 \mathrm{~nm})$, the intermediate band width, $\Delta_{1}$, with QD width at different values of barrier width is considered in Figure 8 . When comparing this figure with the previous Figure 6, they gave the same trend. On the other hand, the varying $\Delta_{1}$ in the first range covers a longer range of energy in comparison with the second one. Moreover, the allowable barrier width range in this second range is shifted left to be from $1.98 \mathrm{~nm}$ to $2.7 \mathrm{~nm}$. The energy gap between the IB and each of VB and $C B$ is illustrated in Figures 9(a) and 9(b). As a result of changing the values of $\Delta_{1}$, the corresponding values of energy gaps will be changed for the composition of the model, QD width $\left(\operatorname{InAs}_{0.9} \mathrm{~N}_{0.1}\right)$ and barrier width $\left(\mathrm{GaAs}_{0.98} \mathrm{Sb}_{0.02}\right)$. Also the curves in this case tend to be straight in comparison with their equivalent in Figures 7(a) and 7(b). One can notice that the second range investigations were not considered before in previous studies. Although it gives an enhancement of power conversion efficiency as shown in the next results.

An example of the $J-V$ characteristics for the second range of the proposed model is mentioned in Figure 10. It is important to note that the short circuit current density is related to the quantum dot width directly; meanwhile the open circuit voltage is approximately constant. Additionally, the wide range of the $J-V$ characteristics with short circuit current and open circuit voltage values is confirmed in Table 2. This will contribute to the enhancement of obtained power conversion efficiency as shown in Figure 11. One can notice that the behavior of efficiency is in full agreement with the obtained results in [25]. But the new contribution is that the efficiency in this case, full concentration, reached up to (70.4\%) (see also Table 2). The observed enhancement of the efficiency returns to discovering second range. Comparison between $J-V$ characteristics for fully and unconcentrated cases are described in Figure 12. From this figure and Table 2, one can recognize that the second range denotes higher values of $J_{\mathrm{sc}}$ and a small amount of increase of $V_{\mathrm{oc}}$ in comparison to 


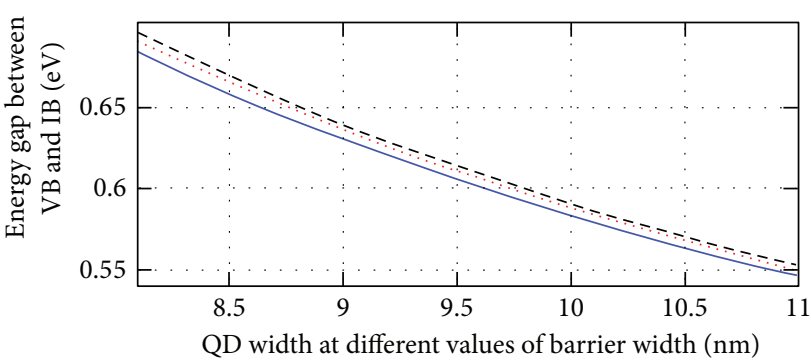

Barrier width:

$\begin{array}{ll}\ldots--1.98(\mathrm{~nm}) \\ \ldots \ldots . & 2.34(\mathrm{~nm}) \\ & 2.7(\mathrm{~nm})\end{array}$

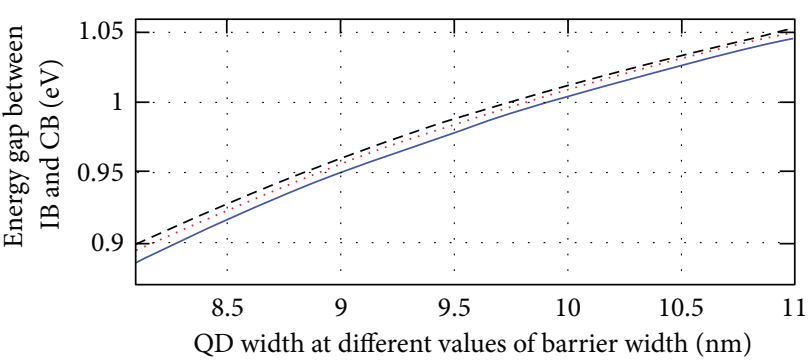

Barrier width:

$--1.98(\mathrm{~nm})$

$2.34(\mathrm{~nm})$

$2.7(\mathrm{~nm})$

(a)

(b)

FIGURE 9: (a) Change energy gap between VB and IB according to change in QD width for the second allowable range at different values of barrier width. (b) Change energy gap between IB and CB with the same conditions in (a).

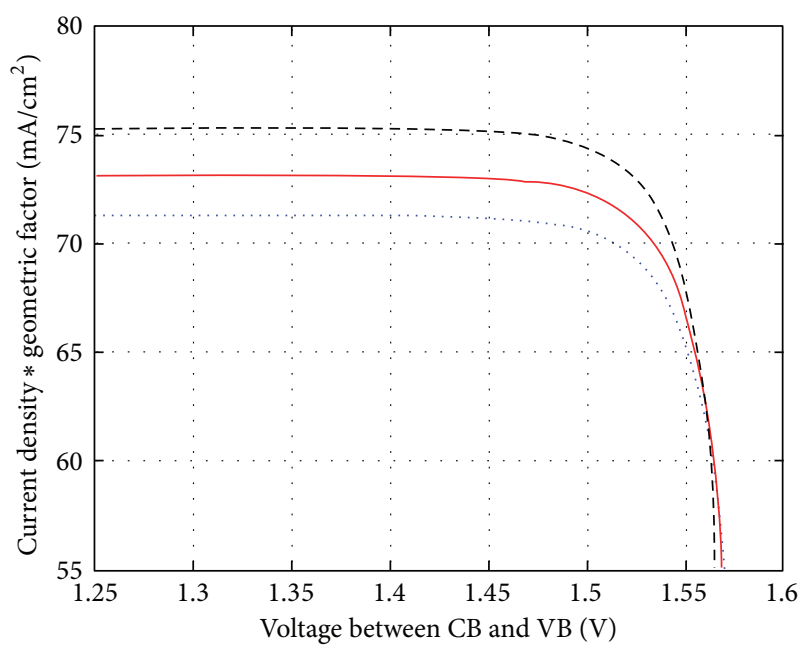

$\mathrm{QD}\left(\mathrm{InAs}_{0.9} \mathrm{~N}_{0.1}\right)=$
$---8.1 \mathrm{~nm}$
$-8.6 \mathrm{~nm}$
$\ldots \ldots .1 \mathrm{~nm}$
$\mathrm{~B}\left(\mathrm{GaAs}_{0.98} \mathrm{Sb}_{0.02}\right)=1.98 \mathrm{~nm}$

FIGURE 10: Current density for QDIBSC at full concentration with varying the quantum dot width and barrier width held constant.

the first range. But in the two considered ranges, the values of $V_{\mathrm{oc}}$ in the full concentration case are higher than that in the unconcentrated case. From a solar cell point of view, this behavior normally happens because the allowable large width of quantum dots will acquire high photons and it then excites a large number of electrons: high induced current density. For the same cases considered before, the comparison between the power conversion efficiency is depicted in Figure 13. Also, the corresponding power conversion efficiency, $\eta, J_{\max }, V_{\max }$, $J_{\mathrm{sc}}, V_{\mathrm{oc}}$, and fill factor, $F F$, for each combination of QD width $\left(L_{\mathrm{QD}}\right)$ and barrier width $\left(L_{B}\right)$ for fully and unconcentration are considered in Table 2 . The fill factor pointed in the table

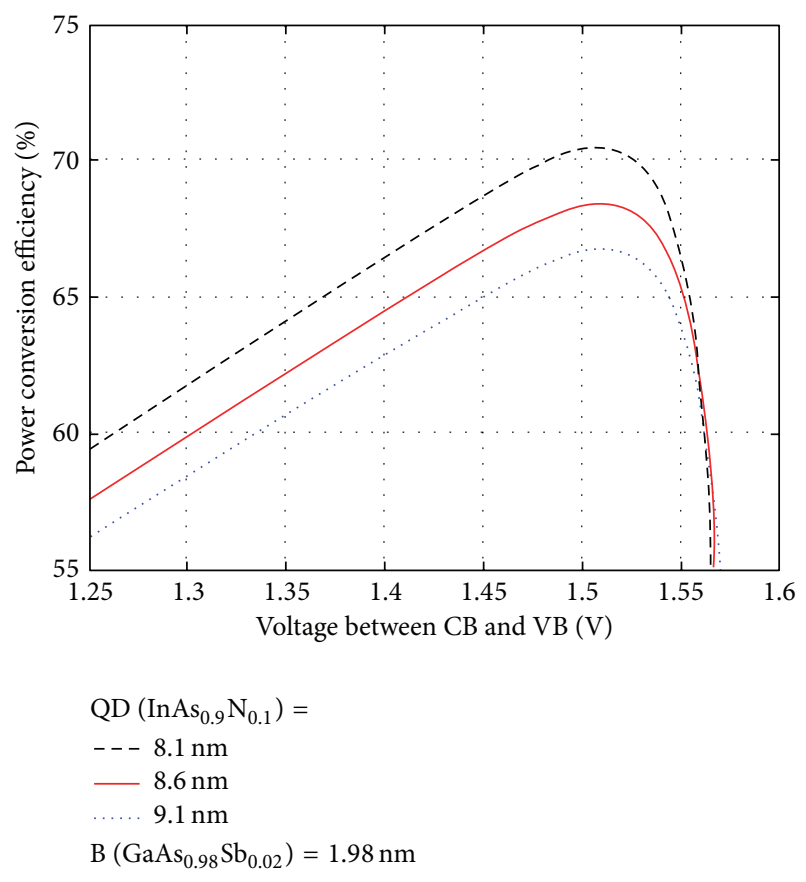

FIGURE 11: Power conversion efficiency for QDIBSC at full concentration with varying the quantum dot width and barrier width held constant.

is determined from (13). It also indicates an enhancement of power conversion efficiency, where $F F$ is greater than $93 \%$ and $90 \%$ in the considered cases, respectively. The main target of these demonstrations is to compare the obtained power conversion efficiency from the first and second ranges. When a full concentration case is taken in the theoretical calculation, higher values of each of the open circuit voltage and short circuit current density are obtained that correspondingly gave higher power conversion efficiency. When one held comparison between our obtained results and others in the fully and unconcentrated cases: $\eta_{\max }=70.4 \%$ 


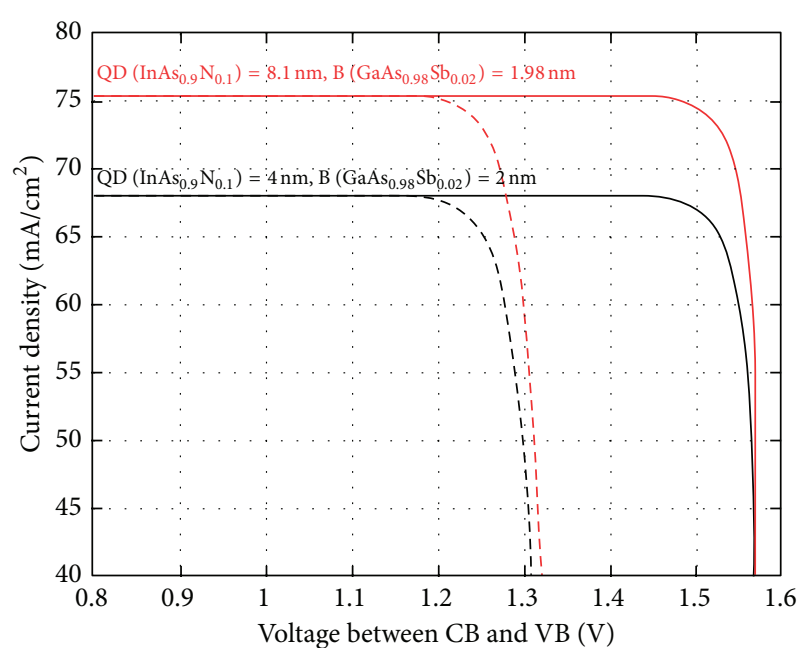

FIGURE 12: Comparison between current density for QDIBSC at full concentration (solid) and unconcentrated (dash) cases at specified values of quantum dot and barrier widths that give maximum efficiency.

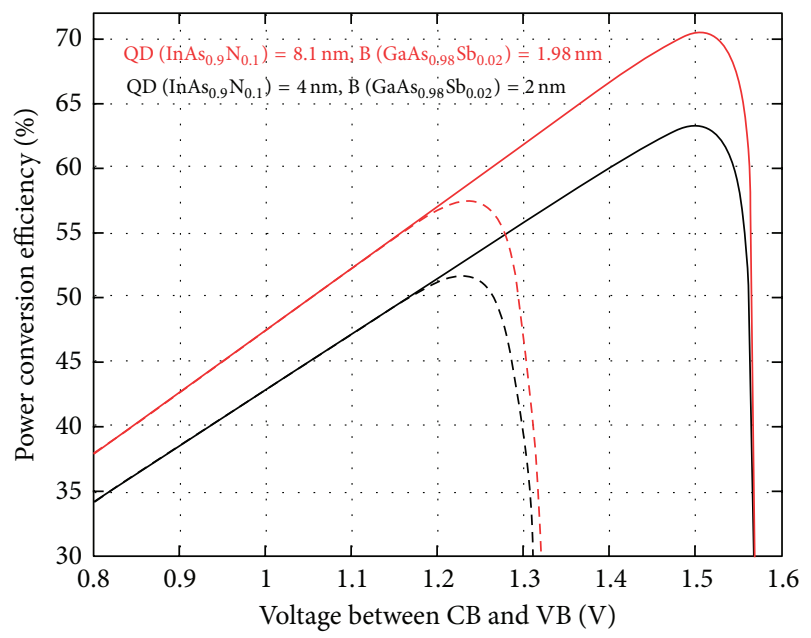

Figure 13: Power conversion efficiency for QDIBSC at full concentration (solid) and unconcentrated (dash) cases at specified values of quantum dot and barrier widths that give maximum efficiency.

rather than $63.2 \%[11,14,26,27]$, and $\eta_{\max }=57.5 \%$ rather than of $46.8 \%$, respectively, [14]. The numerical results in Table 2 can be utilized also for experimental achievements. In further investigations, the effect of two intermediate bands for different alloys into the power conversion efficiency will be studied.

\section{Conclusion}

In this work, a theoretical study for the QDIBSC that would contain one intermediate band between the valence and conduction bands was investigated. This intermediate band exploits the low energy photons to enhance the transferring process of charge carriers from valence to conduction bands. Otherwise, it would normally be lost as heat or not absorbed by the conventional solar cell. As a sequence of utilizing the proposed model, the incoming photons would be well-matched with the energies among bands; thereby the thermalization is reduced. From the obtained results, the open circuit voltage is approximately constant while the short circuit current density is increased. As a result, the fill factor will be enhanced by more than $90 \%$. The power conversion efficiency was calculated under two different ranges of QD width, specified by three different values of barrier width; that is, $L_{\mathrm{OD}}$ from $4-7 \mathrm{~nm}$, at $L_{B}$ values $2,2.5$, and $3 \mathrm{~nm}$ and $L_{\mathrm{QD}}$ from 7-11 nm, at $L_{B}$ values $1.98,2.34$, and $2.7 \mathrm{~nm}$, respectively. The power conversion efficiency is affected by changing the QD and barrier widths. It reaches the maximum value, $63.2 \%$, for full concentration light case in the first range when QD width is $4 \mathrm{~nm}$ and barrier width is $2 \mathrm{~nm}$, which agrees with previous studies. The new facts that have not been addressed before are (a) for the second range, power conversion efficiency reaches $70.4 \%$ when QD width is $8.1 \mathrm{~nm}$ and the barrier width is $1.98 \mathrm{~nm}$ in the case of full concentration and (b) the maximum efficiency for unconcentrated light case is $51.5 \%$ at the first range and $57.5 \%$ for another range. One can recognize from the obtained results an enhancement for efficiency in each of the unconcentrated cases into two ranges and the case of full concentration in the second range. These results of efficiencies create more motivation for further research in this type of structure; therefore future work will concentrate on two intermediate bands: QD solar cell and other alloys with different compositions.

\section{Conflict of Interests}

The authors declare that there is no conflict of interests regarding the publication of this paper.

\section{References}

[1] W. Shockley and H. J. Queisser, "Detailed balance limit of efficiency of $p-n$ junction solar cells," Journal of Applied Physics, vol. 32, no. 3, pp. 510-519, 1961.

[2] A. Martí, E. Antolín, E. Cánovas, N. López, and A. Luque, "Progress in quantum-dot intermediate band solar cell research," in Proceedings of the 21st European Photovoltaic Solar Energy Conference, Dresden, Germany, 2006.

[3] A. Nasr and A. Aly, "Theoretical investigation of some parameters into the behavior of quantum dot solar cells," under publication.

[4] A. Nasr, "Theoretical study of the photocurrent performance into quantum dot solar cells," Optics \& Laser Technology, vol. 48, pp. 135-140, 2013.

[5] A. Luque and A. Martí, "Increasing the efficiency of ideal solar cells by photon induced transitions at intermediate levels," Physical Review Letters, vol. 78, no. 26, pp. 5014-5017, 1997.

[6] A. Luque, P. G. Linares, E. Antolín et al., "Understanding the operation of quantum dot intermediate band solar cells," Journal of Applied Physics, vol. 111, Article ID 044502, 2012.

[7] P. G. Linaresa, C. D. Farmerb, E. Antolína et al., "In ${ }_{\mathrm{x}}\left(\mathrm{Ga}_{\mathrm{y}} \mathrm{Al}_{1-\mathrm{y}}\right)_{1-\mathrm{x}}$ As quatemary alloys for quantum dot intermediate band solar cells," Energy Procedia, vol. 2, no. 1, pp. 133-141, 2009. 
[8] C. - Lin, W.-L. Liu, and C.-Y. Shih, "Detailed balance model for intermediate band solar cells with photon conservation," in Proceedings of the 37th IEEE Photovoltaic Specialists Conference (PVSC '11), 2011.

[9] L. Cuadra, A. Martí, and A. Luque, "Present status of intermediate band solar cell research," Thin Solid Films, vol. 451-452, pp. 593-599, 2004.

[10] M. Y. Levy, C. Honsberg, A. Marti, and A. Luque, "Quantum dot intermediate band solar cell material systems with negligable valence band offsets," in Proceedings of the 31st IEEE Photovoltaic Specialists Conference, pp. 90-93, January 2005.

[11] Q. Deng, X. Wang, C. Yang et al., "Theoretical study on $\mathrm{In}_{\mathrm{x}} \mathrm{Ga}_{1-\mathrm{x}} \mathrm{N} / \mathrm{GaN}$ quantum dots solar cell," Physica B: Condensed Matter, vol. 406, no. 1, pp. 73-76, 2011.

[12] O. L. Lazarenkova and A. A. Balandin, "Miniband formation in a quantum dot crystal," Journal of Applied Physics, vol. 89, no. 10, pp. 5509-5515, 2001.

[13] Q. Shao, A. A. Balandin, A. I. Fedoseyev, and M. Turowski, "Intermediate-band solar cells based on quantum dot supracrystals," Applied Physics Letters, vol. 91, no. 16, Article ID 163503, 2007.

[14] E. J. Steven, Quantum dot intermediate band solar cells: design criteria and optimal materials [Ph.D. thesis], Drexel University, Philadelphia, Pa, USA, 2012.

[15] T. Soga, Nanostructured Materials for Solar Energy Conversion, 1st edition, 2006.

[16] S. Birner, Modeling of Semiconductor Nanostructures and Semiconductor-Electrolyte Interfaces, 2011.

[17] S. P. Day, H. Zhou, and D. L. Pulfrey, "The Kronig-Penney approximation: may it live on," IEEE Transactions on Education, vol. 33, no. 4, pp. 355-358, 1990.

[18] R. Aguinaldo, Modeling solutions and simulations for advanced III-V photovoltaics based on nanostructures [M.S. thesis in materials science \& engineering], College of Science, Rochester Institute of Technology, Rochester, NY, USA, 2008.

[19] W. van Roosbroeck and W. Shockley, "Photon-radiative recombination of electrons and holes in germanium," Physical Review, vol. 94, no. 6, pp. 1558-1560, 1954.

[20] A. Martí, L. Cuadra, and A. Luque, "Quasi-drift diffusion model for the quantum dot intermediate band solar cell," IEEE Transactions on Electron Devices, vol. 49, no. 9, pp. 1632-1639, 2002.

[21] L. A. Kosyachenko, Solar Cells-New Aspects and Solutions, 2011.

[22] S. P. Bremner and C. B. Honsberg, "Intermediate band solar cell with non-ideal band structure under AMl.5 spectrum," in Proceedings of the 38th IEEE Photovoltaic Specialists Conference (PVSC '12), 2012.

[23] A. Martí, L. Cuadra, and A. Luque, "Partial filling of a quantum dot intermediate band for solar cells," IEEE Transactions on Electron Devices, vol. 48, no. 10, pp. 2394-2399, 2001.

[24] J. Ojajärvi, Tetrahedral chalcopyrite quantum dots in solar-cell applications [M.S. thesis], Department of Physics, University of Jyväskylä, 2010.

[25] A. Luque, A. Martí, and L. Cuadra, "Impact-ionization-assisted intermediate band solar cell," IEEE Transactions on Electron Devices, vol. 50, no. 2, pp. 447-454, 2003.

[26] Q.-W. Deng, X.-L. Wang, C.-B. Yang et al., "Computational investigation of $\operatorname{In}_{\mathrm{x}} \mathrm{Ga}_{1-\mathrm{x}} \mathrm{N} / \mathrm{InN}$ quantum-dot intermediateband solar cell," Chinese Physics Letters, vol. 28, no. 1, Article ID 018401, 2011.
[27] A. Nasr, "Theoretical model for observation of the conversion efficiency into quantumdot solar cells," under publication. 

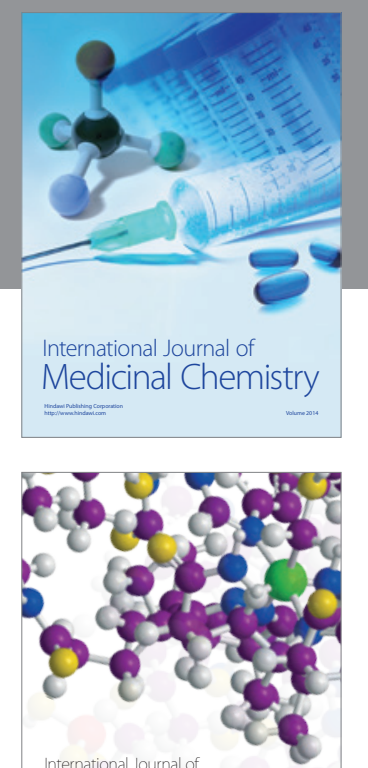

\section{Carbohydrate} Chemistry

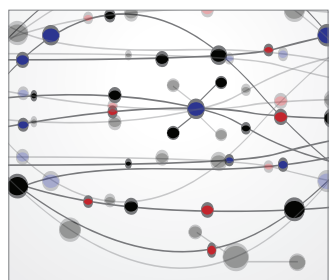

The Scientific World Journal
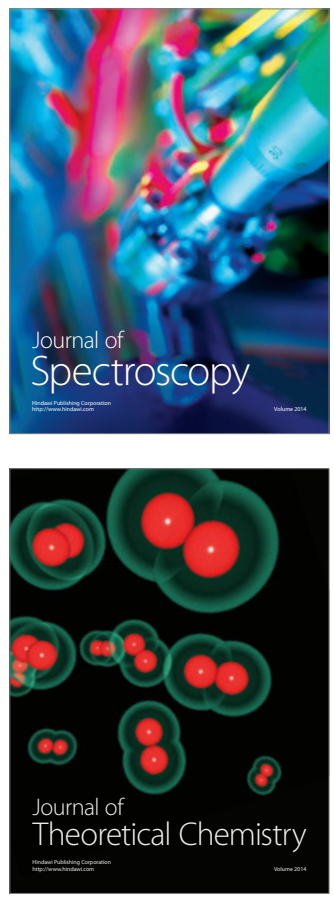
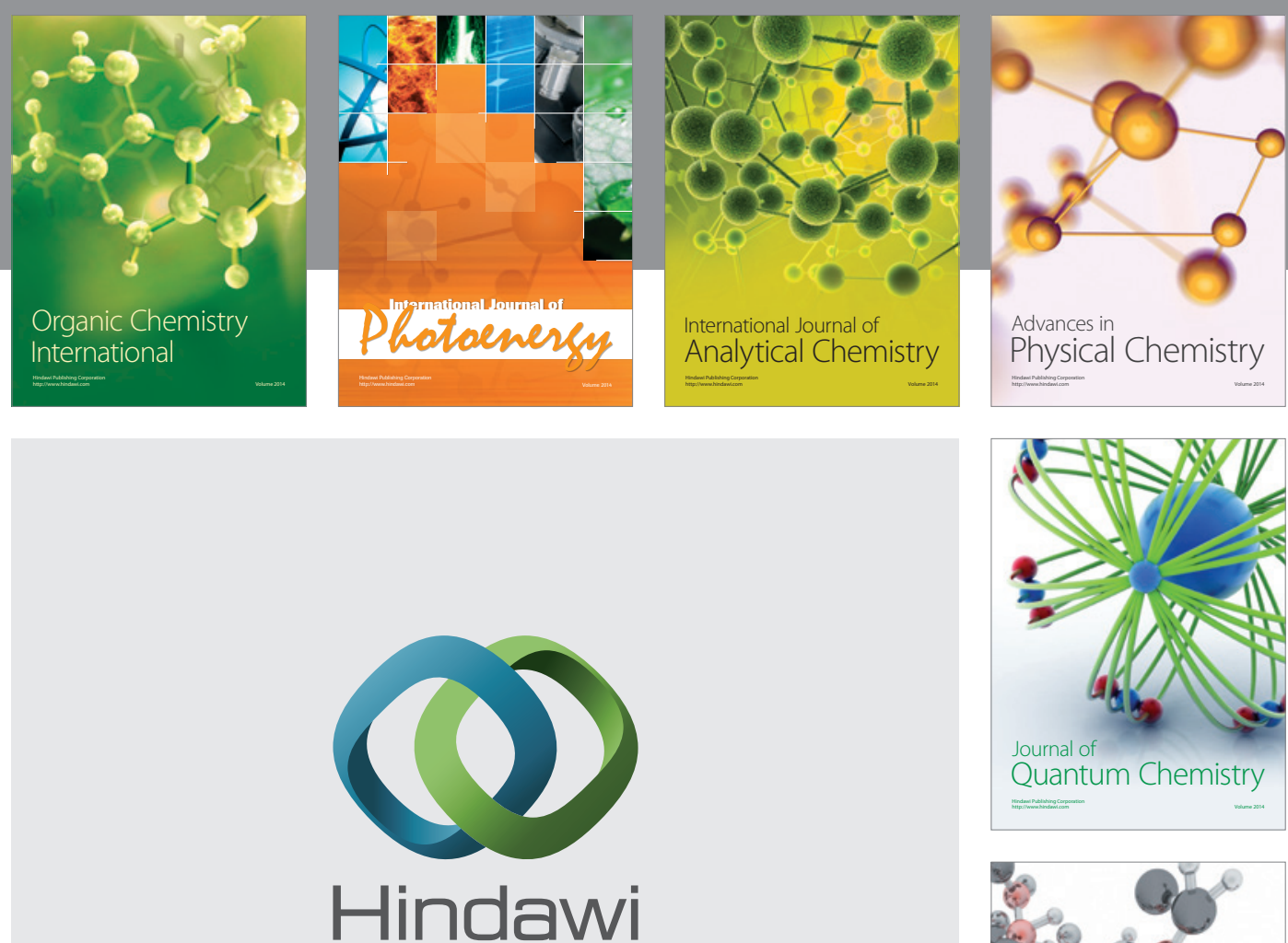

Submit your manuscripts at

http://www.hindawi.com

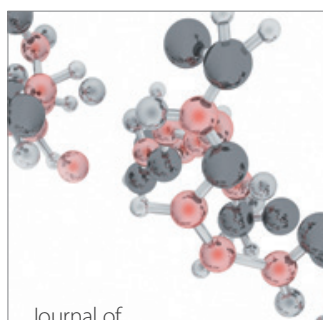

Analytical Methods

in Chemistry

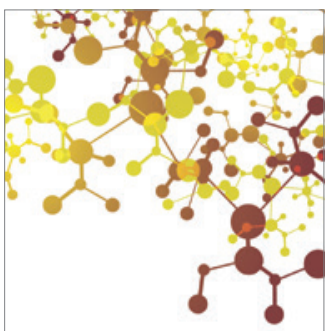

Journal of

Applied Chemistry

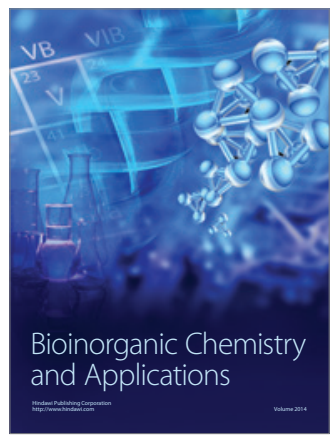

Inorganic Chemistry
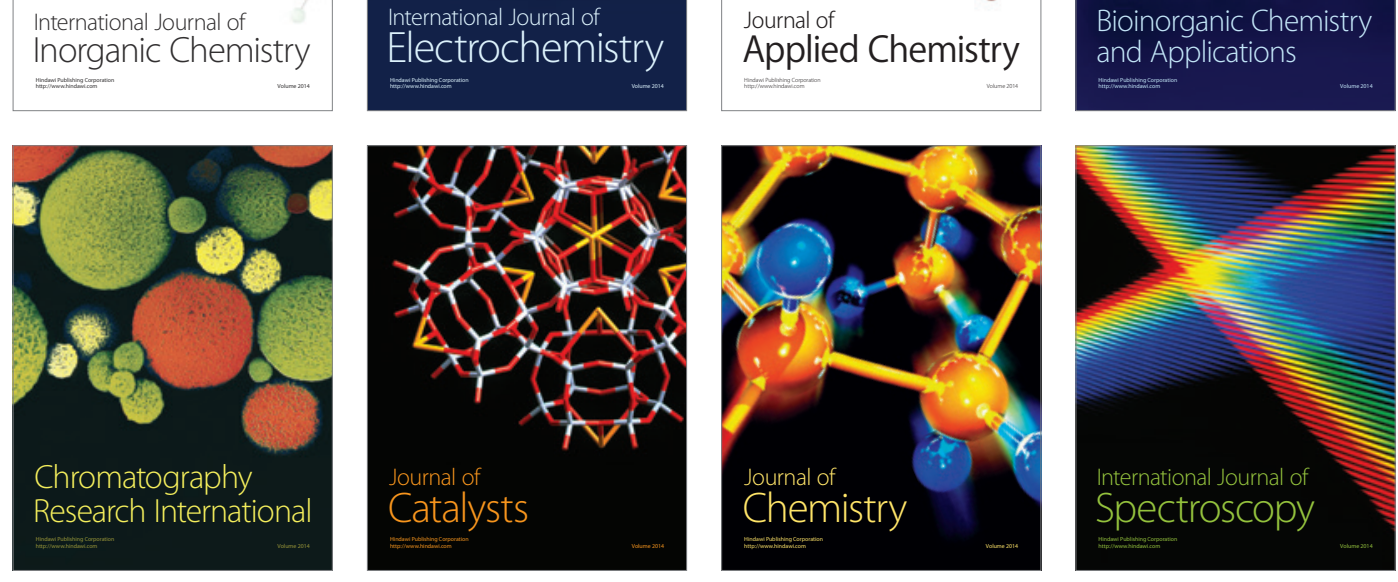